\title{
Evolution of SARS-CoV-2 mutational variants: Hysteria or hope for the future?
}

For almost 2 years, the coronavirus disease 2019 (COVID-19) pandemic has continued its devastating impact across the world. As of December 2021, there have been more than 270 million confirmed cases globally, including over 5 million deaths. Severe acute respiratory syndrome coronavirus 2 (SARS-CoV-2), similar to other ribonucleic acid (RNA) viruses, is prone to adaptative genetic evolution and mutations, resulting in the emergence of new variants with varied clinical characteristics. ${ }^{[1]}$ At least five variants of SARS-CoV-2, the virus that causes COVID-19, have been classified as variants of concern (VOCs). ${ }^{[2]}$ The Alpha variant (B.1.1.7) and the Beta variant (B.1.351/501Y.V2) were first documented in September 2020 in the UK and South Africa (SA), respectively, while the Gamma variant (P.1/501Y.V3) was reported in Brazil in October 2020. The Delta variant (B.1.617.2/478K.V1) was first detected in India in December 2020 and spread to at least 40 countries, thus becoming the globally dominant variant. The most recent VOC, the Omicron variant (B.1.1.529), was documented in several countries, including SA, in November 2021. While robust epidemiological data have provided evidence for the increased transmissibility of these VOCs, each more than the last, their impact on clinical outcomes such as disease severity and mortality in specific populations is less clear. Thus, studies looking at clinical correlations of VOCs are important in understanding disease severity and assist us in understanding the trajectory of the pandemic, which in turn allows for the planning of resources for future waves.

In this issue of the AJTCCM, Lalla et al. ${ }^{[3]}$ add to a body of research by describing and comparing clinical characteristics, management, and patient outcomes, including mortality and length of stay, in an intensive care unit (ICU) in SA during the first and second COVID-19 waves. A total of 490 patients were admitted to the ICU, including 408 from the first wave and 82 from the second wave, with both groups being similar regarding risk factors associated with poor outcomes. Notwithstanding study limitations, which are acknowledged in the publication, the key finding of this study was that there was no observed difference in mortality between the first and second waves $(62.5 \%$ v. $65.9 \%)$. However, the rates of mechanical ventilation $(14.0 \%$ v. $39.0 \%)$ and, consequently, the duration of ICU stay (6 v. 10 days) was significantly greater in the second wave. These findings demonstrate the severity and devastating impact of the SA second wave, which was dominated by the Beta variant when compared to the first wave which was attributable to the ancestral strain of the novel SARS-CoV-2. This study also demonstrated the evolution of evidence-based strategies for treating patients in ICU, for example, by decreasing routine prescription of empirical antibiotics and adjunctive therapy, such as thiamine and vitamin C. It is often difficult to tease out if the observed severity in disease is attributable to the biological virulence of the VOC or was simply as a consequence of an overburdened health system unable to cope with the sheer volume of patients. Lalla et al. ${ }^{[3]}$ do acknowledge that lack of ICU resources during the second wave may have confounded the findings. Be that as it may, this study still provides insights on the trajectory and future of the pandemic. When the findings of this study are contextualised with the current state of the pandemic driven by the Omicron variant, a picture begins to emerge. Early signs suggest that the Omicron variant may be the most transmissible VOC to date, but seems to be less virulent compared with all other preceding VOCs. $^{[4]}$

SARS-CoV-2 causes more severe disease (five- to six-fold increased risk of ICU admission ${ }^{[5]}$ ) and mutates at a faster rate, ${ }^{[6]}$ compared with influenza. During the 1918 influenza pandemic, most deaths occurred during the first three waves, while a fourth wave (in 1920), was driven by a variant that was much less virulent. This may be very similar to what is being observed currently in the COVID-19 pandemic. Does this apparent decrease in the virulence of SARS-CoV-2 signal the beginning of the end of the COVID-19 pandemic? We hope that history repeats itself (fingers crossed).

Finally, as the virus evolved, so did we. We have several effective vaccines, rapid and efficient vaccine development platforms (such as mRNA vaccine technology), and at least two effective oral agents (molnupiravir and paxlovid) for treatment of mild-to-moderate COVID-19. These, when paired with the resilience demonstrated by frontline clinicians such as Lalla et al. ${ }^{[3]}$ give us hope for our future.

A Esmail, MD, FCP (SA), Cert Pulm (SA)

Centre for Lung Infection and Immunity, Division of Pulmonology, Department of Medicine and UCT Lung Institute, University of Cape Town, South Africa

A J Scott, BSc, MB ChB, MPhil

Centre for Lung Infection and Immunity, Division of Pulmonology, Department of Medicine and UCT Lung Institute,

University of Cape Town, South Africa

K Dheda, MB BCh, FCP (SA), FCCP, PhD (Lond.),

FRCP (Lond.)

Centre for Lung Infection and Immunity, Division of Pulmonology, Department of Medicine and UCT Lung Institute, University of Cape Town, South Africa; South African MRC/UCT Centre for the Study of Antimicrobial Resistance, University of Cape Town, South Africa; Faculty of Infectious and Tropical Diseases, Department of Infection Biology, London School of Hygiene and Tropical Medicine, UK

Afr J Thoracic Crit Care Med 2021;27(4):136-137. https://doi. org/10.7196/AJTCCM.2021.v27i4.204 
1. Aleem A, Samad ABA, Slenker AK. Emerging variants of SARS-CoV-2 and novel therapeutics against coronavirus (COVID-19). In: StatPearls. Treasure Island (FL): StatPearls Publishing, 2021.

2. Pango lineages. Global report investigating novel coronavirus haplotypes. 2021 https://cov-lineages.org/index.html (accessed 20 December 2021)

3. Lalla U, Koegelenberg CFN, Allwood BW, et al. Comparison of patients with severe COVID- 19 admitted to an intensive care unit in South Africa during the first and second wave of the COVID-19 pandemic. Afr J Thoracic Crit Care Med 2021;27(4):156-162. https://doi.org/10.7196/AJTCCM.2021.v27i4.185
4. Abdool Karim SS, Abdool Karim QA. Omicron SARS-CoV-2 variant: A new chapter in the COVID-19 pandemic. Lancet 2021;398(10317):2126-2128.

5. Petersen E, Koopmans M, Go U, et al. Comparing SARS-CoV-2 with SARS-CoV and influenza pandemics. Lancet Infect Dis 2020;e238-244. https://doi.org/10.1016/ S1473-3099(20)30484-9

6. Liu L, Zeng F, Rao J, et al. Comparison of clinical features and outcomes of medically attended COVID-19 and influenza patients in a defined population in the 2020 respiratory virus season. Front Public Health 2021;9:587425. https://doi.org/10.3389/ fpubh.2021.587425 